\title{
V.
}

Aus der psychiatrischen Klinik zu Marburg (Prof. Cramer).

\section{Zur Lehre von der Aphasie.}

\author{
Von \\ K. Cramer, \\ Assistent am Spital za Solothurn.
}

\begin{abstract}
Seit der Aufstellung einer atactis chen (später motorischen) Aphasie, mit ihrer Localisation in der linken unteren Stirnwindung durch B roca und einer sensorischen Aphasie (Aphasie mit Worttaubheit) durch Wernicke, sind zahlreiche Versuche angestellt worden, für die verschiedenen Störungen der Wort- und Schriftsprache und ihre thatsächlichen oder theoretisch möglichen Combinationen an der Hand mebr oder weniger mannigfacher Schemata scharfe Localdiagnosen zu stellen, die nicht immer auf den festen Boden gesicherter anatomischer Kenntnisse von den supponirten Centren und Leitungsbahnen sich gründeten. Besonders wurden mehr als Noth that zum Verständniss complicirender Störungen der Schriftsprache gesonderte Läsionen mit dem Effect der Wortblindheit angenommen, wobei nicht immer gebührend festgehalten wird, dass jede Läsion des Wortbegriffs die „innere Sprache“, in der wir denken, und damit auch die Schriftsprache schädigen muss, während dabei die Schriftsprache soweit sie ohne Verständniss des Geschriebenen möglich ist, erhalten sein kann. Es drohten sogar über diesen, gar zu sehr in's Detail gehenden, Studien jene beiden anatomisch gut gestützten Grundpfeiler der Aphasielehre in's Wanken zu gerathen, als Grashey nachwies, dass es Fälle von Aphasie giebt, in welchen die Störung nicht auf Vernichtung der Bahnen und Centren, sondern darauf beruht, dass die dauernden Sinneseindrücke vermindert sind, also wesentlich auf einer Gedächtnissschwäche. Naunyn*) gebührt das Verdienst, bei voller

*) Naungn, Ueber die Localisation der Aphasie. (Verhandl. des Congr. f. innere Medicin. Wiesbaden 1887.)
\end{abstract}


Anerkennung der fundamentalen Bedeutung dieses durch Grashey eingeführten Moments, aus einer umfangreichen und in sich sehr ungleichwerthigen Literatur das herausgeschält zu haben, was die Wissenschaft in der Frage der Localisation der corticalen Aphasie bisher als wohlbegründeten und bleibenden Besitz ihr eigen nennt.

Unter 71 von ihm gesammelten guten Krankengeschichten mit Sectionsbefund, wo Läsionen in Hirnwindungen selbst oder unmittelbar darunter lagen, befinden sich 24 rein motorische, 18 rein sensorische, 36 unbestimmte Aphasien. Unter den 24 motorischen befand sich kein einziger, wo nicht die Broca'sche Windung, unter den 18 sensorischen kein einziger, wo nieht die Wernicke'sche Windung lädirt war. Die 36 Fälle unbestimmter Aphasie neigten sich bald mehr zur motorischen, bald mehr zur sensorischen; dementsprechend fand sich in $60 \mathrm{pCt}$. derselben eine Läsion der Broca'schen oder der Wernicke'schen Windung, in $20 \mathrm{pCt}$. eine Läsion in so grosser Nachbarschaft einer dieser beiden Windungen (Insel, mittlere Stirnwindung, G. marginalis), dass eine Mitstörung derselben anzunehmen ist, in den restirenden $20 \mathrm{pCt}$. findet sich als Localisation die Gegend, wo der G. angularis in den Lob. occipitalis übergeht. Weitere Untersuchungen werden festzustellen haben, ob diese Stelle, welche den mit Hemianopsie verknüpften Herden sehr nahe liegt, vielleicht sich mit der Localisation der optischen Aphasie Freund's (Archiv für Psych. XX., 1 and 2) deckt, welcher unter dieser Bezeichnung ein immer mit Gesichtsfelddefecten verknüpftes Symptom heraushebt, das in der Schwierigkeit, vorgezeigte Gegenstände zu benennen, besteht. Dies Symptom, das auch in dem Wernicke'schen Krankheitsbilde der subcorticalen Alexie sich wiederfindet, setzt eine Unterbrechung zwischen optischer Componente des Gegenstandsbegriffs und Wortbegriffs voraus, die nach unseren bisherigen Kenntnissen in jener Gegend zu suchen wäre.

„Diese drei Rindenfelder", schliesst Naunyn, indem er ihre höchst bedeutenden Beziehungen zu den Centren der motorischen und sensorischen Vorgänge, welche die wesentlichste Rolle beim Sprechen spielen, darthut, „sind nicht Sprachcentra, sondern sie stellen lediglich die Stellen der Rinde dar, von denen aus nachdrückliche Störungen dieses complicirten Mechanismus am sichersten herrorgerufen werden können".

Diese Formulirung muss als eine höchst glückliche und für alle Rindenfelder zutreffende bezeichnet werden.

Exner und Paneth haben für die motorische Region experimentell dargethan, was bis dahin nur theoretisch postulirt werden musste, 
dass Umschneidung eines Rindenfeldes dasselbe lahm legt. Wäbrend es durch äussere Reize z. B. durch den elektrischen Strom erregbar bleibt, fehlt jede Spontaneität der Bewegung, da mit der Unterbrechung aller Associationsbahnen zu dem Centrum jegliche Erregung durch innere Reize (Vorstellungen) wegfällt. Vorstellungen der verschiedensten Dignität (optische, acustische, tactile etc. Vorstellungsreihen in den mannigfachsten Combinationen) sind es, welche als Reize auf den verschiedensten Bahnen an die Rindenfelder, auch die der Sprache, herantreten, sie zur Thätigkeit veranlassen. Bei functioneller oder anatomischer Isolirung nacb einer Seite hin kann unter Umständen die Innervation noch von anderer Seite her mit Erfolg übernommen werden. Der Functionsausfall wird um so merklicher, je mehr die Isolirung fortschreitet und je bedeutsamer die Rolle ist, welche die unterbrochene Bahn bei der Innervation bisher spielte; totale Isolirung oder Vernichtung der corticalen Sammelstellen (hier für die Componenten des Wortbegriffs) wird am sichersten nachdrückliche Störungen der Function zur Folge haben.

Es ist gewiss recht auffallend, dass $\mathrm{Naunyn}$ unter den vielen Hunderten von Fällen, die er der Durchsicht unterwarf, nur 71 für seine $\mathrm{Zwecke}$ brauchbare, d. b. klinisch und anatomisch genügend beschriebene fand, darunter nur 18 von sensorischer Aphasie. Er selbst fordert auf zu einer ferneren Sammlung verwerthbaren Materials beizutragen. Icb folgte daher gern der Anregung des Herrn Prof. Cramer, einen in der Marburger psychiatrischen Klinik klinisch und anatomisch genau bearbeiteten Fall von sensorischer Aphasie mitzutheilen.

Oberförster A. ans Treissbach, geboren 1822 (rec. 10. Juni 1884, gestorben 14. December 1888). Es liegt keine hereditäre Belastung vor. Der Vater ist an einer Magenerkrankung, die Mutter an Altersschwäche gestorben. Patient entwickelte sich körperlich und geistig normal. Im 30. Lebensjabre überstand er den Typhus. Sonst soll er bisher immer gesund gewesen sein. In seinem Dienste war er früher immer sehr tüchtig. In den letzten Jahren dagegen wurde eine schlechte Geschäftsführung bemerkt, so dass er sich Tadel and Geldbussen von Seiten der Regierung zuzog. Er fiel dann auch durch sein menschenschenes, still zurückgezogenes Wesen auf. Selbst mit seinen Angehörigen verkehrte er wenig. Seine Gesnndheit hütete er ängstlich, so ass er Abends nur sehr wenig, weil or sonst schlecht zu schlafen glaubte. Geistigen Getränken war er abbold. In seiner Gegend galt er schon seit mehreren Jahren als ein "verdrehter Herr". In seinem Zimmer durfte Niemand etwas anrühren. Wie sich später bei einer gerichtlichen Inventaraufnahme herausstellte, herrsohte in seiner Wobnung und besonders in den Schubladen grosse Unordnung. Seine Coupons hatte er schon lange nicht mehr abgeschnitten. 
Ferner fand man dienstliche Schriftstücke, werthlose Papiere, Acten etc. otc. bunt durcheinander liegen mit Silber- und Goldstüeken und Werthpapieren im Betrage von im Ganzen 28,000 Mk.

Am 9. Juni 1884 Morgens beim Kaffeotrinken klagte Patient seiner Nichte, es sei inm wunderlich mit der Sprache, er wisse gar nicht, wie jhm sei. Dann fing er an verkehrt zu reden, pfiff, lief umber und war sehr unruhig, sprach von Gift, das ihm die Nichto in den Kaffeo gethan. Mittags ass er mebrere Teller Suppe. Abends gegen $6 \mathrm{Uhr}$ soll ein kaum eine Minute andauernder apoplectiformer Anfall aufgetreten sein, bestehend in Zuckungen des Gesichts, der Arme und Beine. Vollständige Bewusstlosigkeit war nicht vorhanden. Lähmungen bestanden nicht, auch nicht der Sprache. Patient konnte sogleich wieder gehen und die Arme gebrauchen. Die rechte Gesichtshälfte soll etwas gehangen haben. Die Benommenheit hielt an; die darauf folgende ganze Nacht hindurch war Patient dann sehr unruhig, delirirte, schlug um sich, lief auf einmal in sein Zimmer, riss eine Flinte von der Wand und wollte anf seine Umgebung schiessen. Er schlug jegliche Nahrung aus. Er warde mit Blutentziehungen hinter den Ohren und Morphium subeutan behandelt. Am 10. Morgens traf ihn der behandelnde Arzt in so aufgeregtem Zustande, dass eine genauere körperliche Untersuchung nicht vorgønommen werden konnte, doch fand er an der Sprache nichts Auffallendes. Die Zunge wurde gerade und obne Zittern vorgestreckt. Im Laufe desselben Tages erfolgte dann die Aufnahmo des Patienten in dio Marbarger Irrenheilanstalt.

Es besteht Aphasie in später zu schildernder Form. Zn Bett gebracht, trinkt er ein Glas Wein und ein halbes Glas Bier, orbricht $3 / 4$ Stunden später und schläft bald ein. Schlief bis zum Morgen des 11. Juni ununterbrochen, wachte dann drei Stunden und schJief darauf weiter; dabei schnarchendes, angestrengtes Athmen. Nach dem Erwachen benommen und unwirsch. Wehrte bei Versuchen, ihm Nahrung zu reichen, eine körperliche Untersuchung vorzunehmen, ab. Nimmt nur wenig zu sich, führt verwirrte und schwer verständlich $\theta$ Reden.

12. Juni. Nach langen Schlaf helit sich das Bewusstsein allmälig auf. Die nunmehr vorgenommene körperliche Untersuchung ergiebt folgenden

\section{Status praesens.}

A. ist ein grosser, kräftig gebauter Mann. Körpergewioht 200 Pfund. Radialarterien sind ein wenig rigid. Am Herzen ist nichts Abnormes zu finden. Lungengrenzen nach abwärts verschoben (mässiges Emphysem). Der Urin ist sauer; spec. Gewicht 1017. Er enthält weder Albumen noch Saccharum. Die Pupillen von mittlerer Weite, reagiren gut reflectorisch wie consensuell. Auch unterscheidet Patient die Farben gat von einander. Patient ist Presbyop; es besteht weder Amblyopie noch Hemianopsie. Die Hörfähigkeit ist vermindert. Flüstersprache bört er rechts auf $80 \mathrm{Ctm}$., links auf $40 \mathrm{Ctm}$. Entfernung. Die Uhr hört er rechts im Contact, links auch da nicht. Beide Trommelfelle sind leicht getrübt and eingezogen. Die Sensibilität ist intact. Die Zunge wird gerade hervorgestreckt. Der rechte Wangen- und Mundfacialis 
ist paretisch. An den Extremitäten keinerlei motorische Lähmung; Patient steht und geht ohne Unterstützung. An den Händen bestellt Tremor. Das Kniephänomen ist beiderseits in normaler Stärke vobanden. Keine Störungen von Seiten der Blase und des Mastdarms. Das weitere psychische Verhalten wird weiter unten besprochen werden. Hier seien zunächst die uns vor Allem interessirenden aphasischen Störungen gescbildert, wie sie sich nach Aufhellung des Bewusstseins bei sehr häufigen, an verschiedenen Tagen wiederholten Untersuchungen herausstellten.

\section{Störungen der Lautsprache.}

1) Beim spontanen Sprechen documentirt sich zunächst eine Verarmung des Wortschatzes, besonders an Hauptworten, speciell Eigennamen, ferner paraphasische Störungen.

12. Juni. „Das ist nun die dritte Stelle, und die anderen sind gegangen und da bin ich nicht dagewesen. Und das können sie nicht erklären, und ist nur einmal gekommen und da ist der - (sucht nach dem Namen) Mann der - - - . Und da ist der eine (ebenso) der hat ja kein, und der Andere, nun der findet, wie kann aber so ein _ _ - (wie oben). Sie haben also — ich weiss selber nicht, wio das ist _ _ es sind also bloss $4 \ldots$ - einmal und die Anderen - jetzt das 3. - - ".

Patient ist offenbar ganz unorientirt über seine Situation; er scicht nach Aufklärung, versteht aber nur sehr unvollkommen, was man ihm sagt und hat Mühe die Worte zu finden.

Während dieser Reden ist er in sichtlicher Erregung, agirt viel mit den Armen, überhört vollständig an ibn gerichtete Fragen.

15. Juni. "Warum thun Sie denn das, Sie haben doch bloss eins. Wie geben Sie denn das jeden Augenblick was Anderes. Das kann jch gar nicht verstehen. Sagen Sie mal, wio kann jeh das versteben. Die braucht das gar nicht. Sie konnte einfach abwarten, sie braucht die Karte nicht kommen zu lassen. Den einen, den einen und den Anderen auch nicht, diese Saubaude. Sie glauben ich bätte im Herumgehen schlecht. Sie brauchen nichts. Das ist ganz eigenthümlich, das eine was ich habe behielt ich, bis ich todt. Dann ist fertig. Gott soll mich helfen".

22. Juni. Dentet durch Gesten und Umschreibungen an, dass er wegen Kopfschmerzen kalte Umschläge zu machen wünscht, findet aber das Wort Umschlag nicht.

7. Juli. "Ich hätte sterngern gelesen (soll heissen "gestern gern")".

8. Juli. "Ich bin so erschreckt, so verstimmt. Ich habe solchen Sturm gekriegt, furchtbar. Lasse Sie mich doch stimmen und gehen. Ich bin ... Lassen Sie mich nur lieber sterben - mogen am Tag - - 6 Wochen gar nichts angerührt, und Niemand sprach mit mir ein Wort. Das war Verstäbung".

2) Eine grosse Anzahl von Gegenständen rermag er nicht zu benennen, giebt aber durch Umschreibungen zu vorsteben, dass er ibren Gebrauch kennt. 
12. Juni. (Uhr?) „Ja das ist $1,2,3,4,5,6,7,8,9,10,11,12$. Das sieht man ja“. (Wie heisst denn das Ding?) „Ja das ist, dass ich das nicht kenn". (Hut.) "Ja das ist ja klar, das ist zum" - - (macht die Bewegung des Aufseizens). (Eisenbahnbillet.) "Das ist zum Fahren". Ébenso wenig konnte er bezeichnen: Stock, Sicherheitsnadel, Medaillon, Kneifzange, Aschenbecher. Das Alphabet und die Zahlenreihe kann or bersagen.

3) Es besteht dentlicher Mangel an Wortverständniss. „Was? Was heisst das? Was soll das heissen? Das verstehe ich nicht, diese Aussprache verstehe ich nicht". Derartige Wendungen gebraucht er oft, wenn man langsam, laut and deutlich mit ibm sprach.

4) Vorgesprochene Hauptworte, Bezeichnungen von Gegenständen, die er nicht zu nennen vermag, kann er nicht oder nur foblerhaft nachsprechen.

27. Juni. (Das ist eine Uhr?) „Ja, ja wohl". (Sagen Sie Uhr!) „ja, jawohl"“.

8. Juli. Spricht nach: (an den Rhein) „im Rhein“. (Kneifer) „Knaper, Knape". (Brille) "Dreiband". (Dintenfass) "So Dinte".

10. Juli. (Schlüssel) "Ringel“. (Uhr) "Uhr". (Hut) -

\section{Störungen der Schriftsprache.}

5) Patient liest die einzelnen Buchstaben riohtig, das ganze Wort aber oft falsch.

15. Juni, Liest: (Professor) "Pross".

27. Jani. (Professor) „Pfoss". (Hanptstadt) "Hauptka,-Haupttachte".

7. Juli. (Krieg) "Kirth".

8. Juli. (Kunstrichter) "Kanstrector". (Sturmschritt) "Sturmschwind“.

6) Beim Schreiben lässt er Worte und Buchstaben aus. Er gebraucht falsche Worte, im einzelnen Wort falsche" Buchstaben, die Bildung der einzelne Buchstaben ist richtig, doch sind manche ausgestrichen und corrigirt. Die Handschrift ist flüchtig, er macht viele Kleckso. Er wiederholt sich sebr oft und vernachlässigt den Satzbar, indem er über dem Ende den Anfang des Satzes vergisst.

6. Juni. (An seine Schwester.) „Gott geliebte Menna! Du wirst so gut sein, mich sofort, augenblicklich bei dir einzudrecken, damit ich sofort bei dir ankommen. Also ich bitte dich sofort bei dir einzutreffeu; sofort lässest du mich sofort einzutreffen; auch stellst du sobald als nur meine beiden sämmtlichen Schwestern auch bierher zu kommen denke dir, dass ich kein Zängen rorstellen kann nun seit der Zeit und komm so schnell, was ich noch kommen kann. Auch Gott Hemmelich bin sofort Goliebte schnell es ist Alles so spät (unleserliches Wort) host du für mich. Hilfe! Hilfe! augenblicklich weust du helfen komm so sobnell, sobnell".

(eodem.) "Gutte liebe Minna! Ich bitte mich doch sofort zu mir einzutreffen, und bei mir sofort hierher zu kommen, auch so gut lasse mir meine Geschwister sofort bei hier augenblicklich einzupacken. Gute liebe Geschwester, 
ach der liebe Gott im Himmel mag einzu helfen und za geschehenen zu hülfen. Ioh bin nun seit gez sechs und dreissig Tagen fest eingesperrt und abgesperrt nicht einen Stock an Papier und huffen darf ich eintreffen. Gern hätte ich meine Schreiben und Hülfen gebündel, aber so war ich veranschlossen als ein verrodert und verdrückter Mensch, wie ein Menschen der gestohlen und unterlassen habe; ich bätte nur einen einzigen einen herufen stellen oder geben dürfen, dann wurde ich angenblicklich, jetzt schon etwas lernen und geken können; es ist mein erstes Pock den ich darfen seit dem sechsten Juli nehmen mit einem kleinen Theilchen den ich darf porstellen dürfte. Liebe Geschwester kommt nur sehr alle zu mir, und helft mir, dass ich Alles auseinander vorstelle und vorsage; Gott liebe Schwester ich könnte sohon mir geholfen werffen was ich dir schreiben konnte, wenn Läde, Papier, Förster, Länder liebe Schwester besorge tofür, dass mir die Hälfen alles voraus vorfielen wird; auch müssen wir für alle gehelfen und pterk Parten guten Enk senken, denn so was ist noch seit Gottes Gedanken und Hersollten nichts poser worden ist; ach gott der liebe Golt wolle sofort helfen dass meine Schwister sofort kommen. Ich bin mir fest eingesperrt und nicht Zeitern und beschreiben. Ich will ja gern schweren wenn ich mir jetzt fern treden kann für meine Schwister. Ich bin stets fest eingesperrt und nun was ich denn vereinstreffen ich mass mich nicht rügen und nur mögte ich fast feststellen, wo wo und was ich gestollen habo; so otwas ist seit Gott krank und kenn sein soll, jeter spürt mich fest und eingesperrt und ist eingetrefft; und mag der Himmel geben, was Gott der Hellest gift; so etwas ist noch nichts gekommt, was geschürt und nun liebe Geschwester riefe mir alles umber, für mich und Hülfe die ganzen Hallen. Der Gottes Hülfe mag einscheren und die ganze Kenntnen seit meines und nur sorge, dass ulle Müetern der Koller könnte vortreffen, was jeder schon gerne helfen wird".

Heinrich A. (schreibt seinen Namen richtig).

$$
\text { Am 6. Juli "1883“ (sic!) }
$$

7. Juli. "Ich bitte die Herrn Verforren, dass ich sofort nach meinen Geschwestern abgeholt und sofort hierher verfarrt werden muss. Auch bitte jeb die Herrn um die Gute dass ich eine Brille sofort hierber geschiokt werden auch für eine Wünder die ich nach Taagstellungen vorgefügt werden kann. Durch die Aussagen kann ich noch nicht wieder darstellen - - - Dio Punotlosungen müssen erst jetzt vielfach dargestellt und ausgesprochen ich muss fast neu ich erden und lernen - - - -

Aehnliche Schriftstücke verfasste er in den nächsten Tagen. Das Alphabet und die Zahlen schreibt or richtig auf.

7) Die Bezeichnung von Gegenständen, die er nicht benenrien kann, kann er auch nicht a ufschreiben.

8) Schreibt auf Dictat mit paragraphischen Störungen $z$. B.

17. Juni (Professor) spricht und schreibt "Pross".

29. Juni (Professor Cramer) "Jorgef".

9) Copirt ganz verkehrt z. B.

7. Juli (Messer) Leber, (Schlüssel) Louge, (Brille) Johann Dache. 


\section{Symptome von Seelenblindheit.}

10) Er erkennt nicht Photographien ron Personen, die ihm bekannt sein mï̈ssen. Das Bild seiner Nichte, die ihm den Haushalt führte, und dio er täglich gesehen hat, jst ihm gänzlich fremd, ebenso eine wohlgelungene Photographie des referirenden Anstaltsarztes. Vorgelegte Münzen verwechselt er. Er hältt z. B. 1 Mark für ein Dreỉmarkstück etc.

Wir fahren nunmehr in der Krankengesehichte fort.

Juni 13. Steht etwas auf. Steht bei der ärztlichen Visite militärisch stramm. Geht in den Garten; betheiligt sich am Kegeln. Weint, dass er die Worte nicht finden kann: "Das ist schlimm, dass ich das nicht nennen kann".

15. Klagt über Kopfweh; spricht von Gift, das ihm eingegeben sein müsse, dass ihm das mit der Sprache so geworden sei. Er ist sehr unwirsch, drängt fort, es sei Unsinn, ihn wegen solcher Kleinigkeit hierher zu bringen.

22. Sehr einsichtslos. Verweigert Arznei (Jodkali) zu nehmen; leicht gerührt und ängstlich, sobald er ein Wort nicht finden kann.

Juli 2. Sebr reizbar; gegen die Aerzte devot, gegen das Wartepersonal gelegentlich grob, verweigert zeitweise die Nahrung.

8. Trägt sich mit Todesgedanken. An dem schlechten Lesen ist die Brille schuld (NB. dieselbe corrigirt seine mässige Presbyopie).

In den nächsten Monaten macht die wahnhafte Verarbeitung des Sprachdefectes im Sinne von Verfolgungsideen weitere Fortschritte. Er würde von allen verḧ̈̈hnt and geärgert. Es würde äber ihn gesprochen, man verstelle und entstelle absichtlich die Worte, damit er sie nicht verstehe. Die Namen würden vertauscht. Er wurde immer misstrauischer, einsilbiger, zurückgezogener, abweisender; er liess sich den Bart nicht schneiden, nahm an Vergnügungen nicht Theil. Das Gedächtniss liess nach. Er beschwerte sich, dass man ibm nichts za lesen gebe, vergass aber ganz, dass fast täglich vergebliche Leseversuche angestellt worden waren.

Die aphasischen Störungen blieben unverändert. Nur wurde der Wortschatz allmälig wieder etwas reicher.

Was den körperlichen Status anbelangt, so ist nachzutragen, dass die Weite der Pupillen wechselte, meist war die rechte weiter wie die linke. Die Parese des rechten Facialis schwand bein Lachen. Zeitweise trat Zittern in den Händen und in der Lippenmuskulatur auf. Das Körpergewicht sank, da der Patient in seinem Verdruss und Unmuth öfters die Nahrung verweigerte.

1885.

Die aphasischen Störungen nahmen an Intensität ab, nur der Mangel an Wortverständniss blieb ziemlich unverändert bestehen. Es stellten sich immer deutlicher Zeichen geistiger Schwäche ein: keine geistigen Interessen, kein Bedürfniss nach irgend welcher Beschäftigung, blöde Beeinträchtigungs- 
ideen. Auch zum Schroibon an seine Familio war er nicht mehr zu bewegen, und liegen aus jener Zeit überbaupt keine spontan verfassten Schriftproben vor.

9. November. Es wird folgendes festgestellt über das jetzige Verhalten der oben aufgefübrten Einzelstörungen. (Nummerirung wie oben.)

1) Spricht spontan mit ziemlich reichem Wortschatz, aber paraphasischen Störungen.

2) Manche der alltäglicheren Gegenstände kann er benennen, z. B. Tintenfass, Messer, Uhr, Bleifeder, Taschentuch, Bier, Fenster, Ofen, Pappelbaum, Schub, Schlüssel; andere dagegen nicht, worüber er selbst klagt, z. B. Aschenbecher, Korkzieber, Ubrkette, Portemonnaie.

3) Der Margel an Wortverständniss, besonders für Hauptwörter ist auffallend; versteht z. B. nicht: "Grossherzogthum Baden", "Darmstadt", "Nassau".

4) Das Nachsprechen geschieht noch fehlerhaft.

$\begin{array}{lc}\text { vorgesprochen } & \text { nachgesprochen } \\ \text { Karlsruhe } & \text { Karlskunge } \\ \text { 29. } 76 & 59.79 \\ \text { 25. } 67 & 25.23 \\ \text { 19. } 38 & \text { (richtig) } \\ \text { 15. } 6 & 15.47 \text { (dann 40) } \\ \text { 32. } 39 & \text { (richtig) } \\ \text { 47. } 53 & \text { (richtig) } \\ \text { 78. } 66 & 78 .(47) .46 . \\ \text { 35. } 47 & \text { (richtig) } \\ \text { 66. } 26 & 66.31(21.25) \\ \text { 46. } 76 & 49.76(47.76) . \\ \text { Politisohe Uebersicht }=\text { Politische Nachricht. } \\ \text { Frankfurter Zeitung } \\ \text { Norddeutsche Allgemeine } \\ \text { Kölnische } \\ \text { Hessische } \\ \text { Deutsches Reich } \\ \text { Rundschau im Auslande }\end{array}$

5) Liest mit Verständniss (?) (beim lauten Lesen parapbasische Störungen); Zahlen, besonders zusammengesetzte, liest er unrichtig.

$\begin{array}{rl}\text { statt } & \text { liest or } \\ 136 & 196(135) \\ 1 & 11 \\ 65 & 57(75) \\ 45 & 47 \\ 34 & 37 \\ 23 & 32 \\ 95 & 97(195) \\ 146 & 376(147,156) \\ 920 & 912(925)\end{array}$




$\begin{array}{ll}\text { statt } & \text { liest } \text { er } \\ 545 & 555(547) \\ 555 & 755 \\ 666 & 565(645,765) \\ 953 & 935 \\ 627 & 677(727,722,637) .\end{array}$

6) $\}$ vacat.

8) Schreiben auf Dictat: "Dor Prinz Heinrich von Preussen verfuhr (soll heissen $f a h r$ ) in der Frühe in Begleitung des Capitain Freiherrn von Weckendorf (s. h. Reckendorf) in der Stadt Springe (s. h. nach Springe) und an der des Kaisers dort Hofjagd tepartement (s. b. um an der rom Kaiser dort abzuhaltenden Hofjagd Theil zu $n \in h m \theta n) "$.

Das Wort „abzuhaltenden " versteht er nicht.

9) Er copirt richtig. Nachfolgendes schreibt er aus einer Zeitung ab: "Nicolai-Markt (Vieh- und Krämermarkt) zu Allendorf a. d. Lomda. Der nächsto Vieh- und Krämermarkt wird in hiesiger Gemeinde Mittwoch den 11. November l. J. abgehalten".

10) Vacat.

11) Nachstehend Rechenproben:

Er zählt zusammon 289

3974

13546

974385

1796195 .

Es wird ihm mündlich das Exempel dictirt:

$$
\begin{array}{r}
4.25 \\
6.36 \\
9.72 \\
8.53 \\
7.29 \\
5.78 \\
3.68 \\
2.47 \\
9.99 \\
12.48 \\
25.54
\end{array}
$$

Er löst es schriftlich folgendermassen:

$$
\begin{array}{r}
4.24=96 \\
6.36=216 \\
4.74=296 \\
8.53=424 \\
7.29=203 \\
5.78=390 \\
3.68=204 \\
2.47=94 \\
9.99=891 \\
12.48=576 \\
25.54=150 .
\end{array}
$$

\section{6.}

Scheues, mürrisohes Verhalten. Es hält schwer, sich mit ihm zu unterhalten, weil or viele Worte falsch versteht und sehr misstrauisch ist. Er isst wenig, weist namentlich Fleiseh zurüok, Nachstehende Notizen aus der, der 
Natur der Sache nach um diese Zeit einförmigen Krankengeschichte bieten einiges Interesse.

7. März. Jaacht mit dem ganzen Gesicht, als er den Hund eines Arztes sieht. Er thaut förmlich auf, streichelt und liebkost den Hund und erzählt von seinen eigenen Hunden. Die Tage darauf wird or dann bäufig gefunden, wie or an der Wand steht und sich unterbält. Er ruft und hetzt Hunde, spricht mit ihnen und macht Bewegungen, als ob er Hunde streichelt, macht Hasenund Rehklagetöne nach etc. etc. Offenbar beschäftigt er sich mit Dingen und Personen aus seinem früheren Leben.

27. Mai. „Es sind lauter Verbrecher hier, deswegen werde ioh hier festgehalten. Sie sind gar kein Arzt. Ich will fort nach Göttingen, dort sind wenigstens Aerzte. Oben (meint den Schlafsaal) bin ich betäubt und das machen Alles diese Herren. Ich höre verkehrte Stimmen. Ioh werde hier geuzt und geneokt, das sind ja gar keine Herren, die hier gehen, die speien mich an, wenn meine Schwestern etwas gemacht haben, so sollen sie vor $\mathrm{Ge}$ richt gestellt werden. Ich will fort. Was hat dann der da (Oberwärter) mich zu prüfen, ob ich roth, weiss etc. unterscheiden kann, ich babe keinem etwas gethan, ich weiss nichts von dem Mord in Witzenhausen".

27. Mai. Schreibt Folgendes: „Herr Professor, sein Sie so gut und lassen Sie mir die Haare nicht schneiden".

20. Juni. Reicht die Hand nicht zum Gruss: „Ach das wissen Sie ja, ich habe schmutzige Hände". Es fehlt jede Krankheitseinsicht: "Ioh bin nicht krank, es giebt nur 7 Farben, keine 8, ich bin auch nicht misstrauisch, ich esse doch. Fleisch esse ich überhaupt nicht, Was sollen denn die armen Menschen essen?" Liest öfters dio Zeitung, versteht auch, was er liest.

15. Juli. "Ich bin doch gar nicht krank. Ich kenne doch die Farben ganz gut". Gefragt, wie es ihm gehe, frägt er selbst: "Wie viel Pinusarten giebts? " Zählt sie dann selbst auf. Wegen zunehmender Nahrungsverweige rung beständige Bettruhe. Von den Extraspeisen, die stets für ihn bereit stehen, rührt er nichts an.

28. Juni. Verwechselt die Personen, redet den Oberwärter mit Dr. Kier, den Herrn Professor mit "Herr Ober-Ober-Medicinalrath" an.

28. August. Spricht mit sich: „Sehr genau, sehr genau. Wir worden uns schon finden. Herr Baron v. Idler, Herr Baron v. Gundlach raus, raus. Westen, Westen, Westen, Westen“. Zum Arzt, der hinzutritt: "Vor Verräthern und Spionen spreche ich nicht. Ich werde hier weggemacht. Da drüben (er meint eine andere Abtheilung) anch schon. Ich habe genau Obacht gewesen; auf einmal war ich ganz weg".

September. Hält die Aerzte für Juristen, versteht sehr oft eine Frage nicht.

October. Hält lange Monologe; sing t a u ch, z. B. "O wie wohl ist mir am Abend" mit richtiger Melodie. Beschwert sich über andere Kranke; so behauptet er von einem neben ihm liegenden ganz stummen and hülflosen Paralytiker, der spräche so viel und laufe wie ein Fuchs. Ist ganz unorientirt, verwechselt die Personen z. B. auch die des Arztes: "Das Gesicht ist wie von Herrn Riehl, das ist kein richtiger Doctor, vielleicht ein Stiehl. Der 
hat mich auf Farben untersucht. Roth, grün, blau. Ich habe geglaubt, dass ich ein Feldmesser. Mit dem Saumensch, mit, der Trapp und Klöckner, da haben sie gebört, das beisst nicht mit der Kley, das bejsst mit der Kleen. Ich habe geglaubt, dass Sie (er meint den besuchenden Arzt) Jurist wären. Und ich glanbe doch, dass Sie ein Jurist sind und das ein sehr geschickter".

November. "Sie sind doch ein Jurist, ich glaube es sicher. So ein Arzit muss 13 Semester haben und muss in Constantinopel und überall gewesen sein. Lassen Sie mich doch nackend nach Hause gehen, ich brauche Niemand". Singt oft ein Lied. Ein Stück aus einem längeren, von einem Wärter nachgeschriebenen Monolog lautet: „Der Soltan verkauft die Eier nur im Winter. Juden und junge Hunde. So eine rerdammte Sauwirthschaft. Schwefelhölzchen, Schwefelholz. So ist es. 4 Pfund Brod sind unbedingt nöthig für einen der Kurfürst pon Hessen. Dor war es gleich zu frieden. Was heisst Schlaf? Das ist nicht nöthig. Ein Arbeiter muss schlafen. Unter dem Lotze ist ein grosser Unterschied. Der eine ging immer mit der Frau, ich glaubs ganz sicher, der hat immer mit ihr getanzt" etc. etc. In dieser Art zieht sich der Monolog noch lange hin.

\section{$188 \%$.}

In den ersten Monaten des Jahres 1887 bleibt sein Zustand unverändert derselbe, wie in der letzten Hälfte des Jahres 1886 . Während der Zeit ron Juni bis October liegt er unbekümmert um die Vorgänge in seiner Umgebung zu Bett und ist nicht dazu zu bewegen, anfzustehen und sich anzuziehen. Er versteht noch sehr schlecht, manche Fragen gar nicht. Spricht manchmal Wochen lang mit Niemanden, hält aber ab und zu Monologe. Ueber seine Umgebung und sich selbst ist er vollständig unorientirt. "Ich bin kein Oberförster, ich bin ein Flegel". Er verificirt seine Träume "Wissen Sie (zum besuchenden Arzte) nichts davon? Mir hat Jemand diese Nacht den Arm abgebrochen. Wer das gewesen ist? " Auf Bemerken des Arztes, dass er das wohl geträumt baben werde, sagt er, 0 nein, die Träume schickt der liebe Gott, der schickt solche Träume nicht". Ferner: "Sie waren diese Nacht" hier; was ist das immer, diese Nacht waren wieder drei hier; der eine habe ich nicht gekannt; der Jäger und der Schafmeister, die müssen sein in Grosseelheim, das wird von dessen eins sein ". Seine Nahrungsaufnahme ist noch immer eine unregelmässige. Zum Waschen ist er nur auf Verlangen des Arztes zu bringen. Er macht dann auf Commando einen Satz aus dem Bette und wäscht sich. Auch ist er jetzt öfters erregt und kommt dann die Aphasie mehr zum Vorschein; z. B. als er seine Unterhosen nicht finden kann: "Wor hat mir denn meine Unterhosen fortgewäsche ${ }^{\alpha}$.

3. December. Frühmorgens hatte er folgenden Anfall: Er machte eigenthümliche Bewegungen mit dem rechten Arm, wurde blau und verlor das Bewusstsein. Der hinzugerufene Arzt findet ihn bewusstlos, sehr erschwert, stertorös athmend, die Augen geöffnet, der rechte Mundwinkel sehr auffällig tiefer stehend. Aus dem Munde fliesst etwas blutiger Speichel. Die Hornhautreflexe sind erhalten. Die Glieder sind schlaff, besonders die rechten Extre- 
mitäten fallen, erhoben, wie ein Fremdkörper herab. Das Kniephänomen ist beiderseits erhalten, der Puls ist gut. Der Anfall dauert etwa $1 / 4$ Stunde. lhm folgt nach einer weiteren $1 / 4$ Stunde ein $z$ weiter, kürzerer Anfall. Danach orholt sich Pationt sehr bald wieder, geht auf den Abtritt, spricht aber nicht und nimmt auch nichts zu sich.

4. December. Patient sagt heute Morgen "ach Gott, ach Gott". Er fängt an zu essen. Lähmungen sind nicht vorhanden.

5. Decomber. Er spricht wieder und weiss, dass er einen Anfall gehabt hat, aber: "Der Anfall ist mir hier gemacht worden, ich bin betäubt worden". Er ass hente ganz ordentlich und ging auf den Abtritt. Die weiteren Tage dieses Monats über zeigt er wieder denselben Zustand wie früher. Er monologisirt viel, so z. B. "Tasso, Tasso, Frankfurter Strasse, hat ein paar Knoblinen geholt, hat aber auch ganz recht gehabt". Er will sich nicht mit dem Kamm kämmen, "Wozu hat uns denn der liebe Gott die fünf Finger gogeben".

\section{8.}

Januar. Patient hält sich noch immer für verfolgt, das Essen hält er für vergiftet; er glaubt, dass man ihn blenden wolle und hält sich deshalb die Hand vor die Augen. Dagegen wäscht or sich jetzt regelmässig, fährt, statt einen Kamm zu benützen, sich mit den Fingern durch's Haar.

12. Februar. Im Betttuch wird ein grosser schmierig-eiteriger Fleck gefunden. Die Untersuchung, gegen welche sich Patient sehr wehrt, ergiebt das Scrotum um das $2-3$ fache vergrössert, die Epidermis macerirt, so dass eine nässende, hier und da schwärzliche Cutis blossliegt. Der Penis ist stark ödematös. Die Haut des Scrotums ist stark infiltrirt, so dass sich über dessen Inhalt nichts Bestimmtes aussagen lässt. Die Nahrungsaufnahme ist sehr gering. Die Temperatur ist leicht erhöht (Patient widerstrebt sehr beim Messen). Es wird ihm ein Sablimatrerband und Suspensorium angelegt; beides zerreisst er jedoch alsbald wieder. Beim Untersuchen wird er böse "habt ihr denn gar keine Soham?"

13. Februar. Die schwärzliche Färbung nimmt za und geht bis übor die Symphyse. Die ganze Haut der Schamgegend ist infiltrirt. Keine Spur von brandigem Geruch. Die Kräfte nehmen zusehends ab. Die Arznei (Aether) wird verweigert.

14. Februar Vormittags 11 Uhr Exitus lotalis.

Sectionsbefund. 15. Februar Vormittags.

Obducent: Pripatdocent Dr. Tuczek.

Todtenstarre gelöst. Todtenflecke auf der vorderen Körperfläche; di Leiche hat auf dem Bauche gelegen.

Rückenmark; Häute normal. In dje Arachnoidea sind einige Knochen- 
plättchen eingelagert. An der Rückenmarkssubstanz auf Querschnitten nichts Auffälliges.

Gehirn: Schädeldach nicht adhärent. Diploe und Tabula vitrea interna und externa in normalem Verhältniss. Im Sinus longitudinalis dunkles Blutgerinnsel. Adrentitialzotten stark gefüllt und prominent. Der Innenfläche der Dura mater liegt über der ganzen Convexität eine dünne pachymeningitische Membran mit sehr entwickeltem, makroskopisch sehr augenfälligem Gefässnetz an. Starkes Oedem der weichen Häute längs der grossen Längsspalte des Hirns. Gewicht des Gehirns mit weichen Häuten $1370 \mathrm{Grm}$. Nach Herausnahme zeigt sich, dass auch die vorderen und mittleren Schädelgruben sowie der clivus Blumenbachii von einer pachymeningitisehen Membran überzogen sind. Die Carotides internae klaffen and sind starrwandig. An der Basilaris wenig Atherom. Die Arteriae fossae sylvii und ihre Verzweigungen sind zartwandig und durchgängig. Pia allenthalben leicht abziehbar. Windungstypus normal. In hohem Grade atrophisch ist nur der Gyrus temporalis superior sinister. Seine Breite beträgt kaum $1 / 3$ der normalen rechten Seite; auch sticht seine gelbbraune Farbe gegen die übrige Rindensubstanz ab. Die Oberfläche der atrophischen Windung ist uneben durch Kämme und narbige Einziehungen. Querschnitte duroh die atrophische linke erste Schläfenwindung ergeben, dass die ganze Markleiste derselben umgewandelt ist in eine braune Narbe mit cystösem Spalt. Diese Voränderung lässt sich verfolgen über die hinteren zwei Drittel der ersten Schläfenwindung und das angrenzende Stück der Marginalwindung, sowie die Uebergangswindung zur Insel. Beim Uebergang der ersten Sohläfenwindung in die Marginalwindung ist die Degeneration der Markleiste, die sich bier noch ein ansehnliches Stück in die Marksubstanz hinab erstreckt (kenntlich durch graue Farbe) am auffälligsten. In der gesammten äbrigen Rinde beider Hemisphären zeigt sich, bei Zerlegung in Querschnitte, keinerlei Herderkrankung; ebenso wenig wie in den Centralganglien. Ventrikel von normaler Weite, Ependym zart. Am Kleinhirn nichts Besonderes. Sämmtliche Venen des Gehirns stark gefüllt. gelegt.

Gehirn und Rückenmark werden zur Härtung in Bichromatlösung ein-

Panniculus adiposus leidlich entwickelt. Muskulatur dürftig. Einige Rippenknorpel verknöchert. Zwerchfellsstand beiderseits an der fünften Rippe. Der Magen ist durch Luft stark ausgedehnt. Im Uebrigen normaler Situs viscerum.

Die Lungen retrahiren sich gut bei Oeffnung der Brustböhle; allseitig frei, nur die rechte Lunge an der hirteren Fläche etwas adhärent. Parenchym durchaus normal.

Herz. Herzbeutel liegt in normaler Ausdehnang vor, enthält klares Serum in gewöhnlicher Menge. Atrioventricularklappen für zwoi Finger durchgängig. Subpericardiales Fettgewebe gut entwickelt. Im rechten Ventrikel wenig dnnkler Cruor. Linker Ventrikel fast leer. Sämmtliehe Klapponapparate intact. Muskulatur des Herzens fest, rothbraun. In der Aorta ascendens 
kaum eine Andentung ron Atherom. Ebenso die Arteriae coronariae zartwandig.

Mil z von normaler Grösse und Beschaffenheit.

Nieren von normaler Grösse. Kapseln leicht abziehbar. Coricalis von gehöriger Breite. Glomeruli deutlich. Der oberen Kuppo der rechten Niere sitzt eine wallnussgrosse Cyste auf.

Leber. Grösse und Beschaffenheit des Parenchyms normal. Gallenblase prall gefüllt mit dunkler, schwerflüssiger Galle.

Magen enthält wenig trübe flässige Speisereste. An der Sobleimhaut nichts Besonderes.

Harnblase enthält ca. 1/4 Liter dunke]n Harnes. Darmcanal zusam. mengefallen, fast leer.

Scrotum: Es ist viel Serum abgelaufen. Haut excoriirt, missfarben; ebenso die des Penis. Unterhantzellgewebe ödematös. Linksseitige Hydrocele, bei deren Eröffnung ca. $100 \mathrm{Cbcm}$. Flüssigkeit ablaufen. Hoden und Nebenhoden normal. Rechts normale Verhältnisse, bis auf einen abgekapselten mandelgrossen Herd, der aussen den Scheiden des Hodens anliegt und dem Anschein nach mit Cholesterinkrystallen gefüllt ist.

Untersuohung des gehärteten Gehirns und Rückenmarks.

Die Zerlegung des Gehirns in Schnitte, welche senkrech auf die Längsaxe des Schläfenlappens geführt wurden, ergab die in Figur I. dargestellte Ausdehnung des Herdes. Derselbe umfasst die I. Schläfenwindung fast ganz,

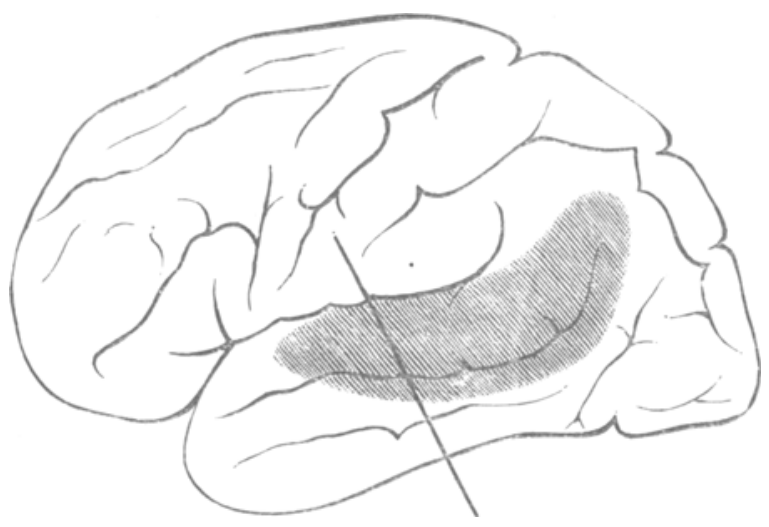

Fig. I.

nur deren Spitze verschonend, die angrenzende Zone der II. Schläfenwindung, und den unteren Abschnitt des Scheitelläppchens, besopders des Gy 
angularis. Wie Fig. II., ein in der Richtung der auf Fig. I. gezogenen Führungslinie gelegter Schnitt zeigt, erstreckt sich der Herd bis auf don Gyrus longus der Insel. In der geschilderten Ausdehnung ist die gesammte Marksubstanz derart zu einer schwieligen Narbe zusammen geschrumpft, dass das stark erweiterte Unterhorn des Seitenventrikels der grauen Rinde sehr nahe kommt. Aus Fig. II., die nach einem Weigertpräparat gezeichnet ist, sieht man, wie der Faserschwund in das Marklager der II. Schläfenwindung und der hinteren Inselwindung incl. äussere Kapsel hineingreift und wie die Markleiste der ersten Schläfenwindung bis auf ein ganz dünnes Lager von Associationsfasern nach der Insel zu ganz von Mark entblösst ist. Die graue Rinde der I. Sehläfenwindung ist stellenweise sehr reducirt und narbig eingezogen; in den benachbarten Windungen, Insel, II. Sohläfenwindung, unteres Soheitelläppchen zeigt sie normale Breite und Contouren. Am ungefärbten Präparat präsentirt sich, bei Betrachtung mit blossem Auge, die Narbe als ein zum

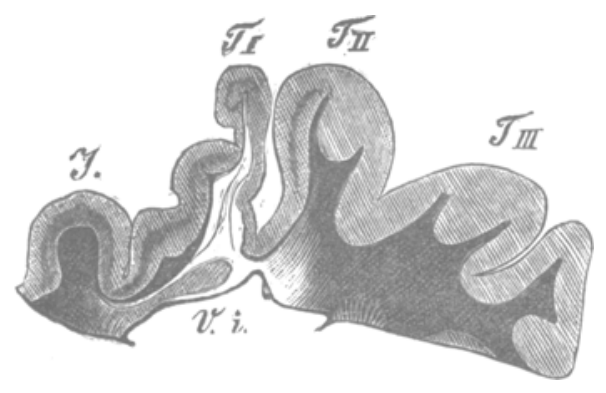

Fig. II. (natürl. Grösse.)

V. i. $=$ erweitertes Unterhorn des Seitenventrikels. J. $=$ Insel.

T I, II, III = erste, zweite, dritte Sehläfenwindung.

Theil weisses, undurchsichtiges (schwieliges) Gewebe, welches rostfarbene Streifen durchziehen und kleine Cysten durchsetzen. Die mikroskopische Untersuchung (Carmin, Boraxcarmin, Weigert'sche Färbung) zeigt als Hauptbestandtheil des Gewebes welliges, an den Grenzen gegen das gesunde Gewebo kernreiches Bindegewebe, mit grossen, zum Theil voluminösen lang ausgezogenen spindelförmigen Zellen. Dies Bindegewebe ist durehsetzt von massenhaftem rostfarbenem Pigment in Körnern and Schollen. Dasselbe ist besonders in der atrophischen I. Schläfenwindung, sowie in einer umschriebenen Partie der II. Schläfenwindung in dichten Zügen (schon makroskopisch sichtbar) angeordnet, dringt aber anch ziemlich weit in die angrenzende normale Marksubstanz ein, wo namentlich die periyasculären Räume davon angefüllt sind. In der grauen Rinde ist das Pigment beschränkt auf den narbigen Antheil der ersten Schläfenwindung. Längs des Unterhorns entspricht die Structur des hier auch die änssere Kapsel und den letzten Ausläufor der Vormauer um- 
fassenden Herdes, nicht gewuchertem Bindegewebe, sondern gewucherter Nouroglia mit erhaltenem Glianetz und reichlichen Spinnenzellen. Das Ependym ist hier verdickt. Die erwähnten kleinen Cysten sind durchzogen von einem zierlichen bindegewebigen Balkenwerk. Das Grundgewebe der narbig eingezogenen verschmälerten grauen Rinde der ersten Schläfenwindung ist zusammengefallenes Gliamaschenwerk; Nervenfasern und Ganglienzellen finden sich darin nioht; in dem die Marksubstanz substituirenden Narbengewebo haben sich nur ganz vereinzelte markhaltige Nervenfasern erhalten. Im Uebrigen erweist sich die graue Rindo der I. Schläfenwindung sowohl wio der angrenzenden Sohläfon- und Inselwindung, sowie des Gyrus marginalis und angularis anch dort, wo die Marksubstanz afficirt ist, als in jeder Beziehung (Ganglienzellen, Reichthum an markhaltigen Nervenfasern, speciell auch in der obersten Schicht) normal. Allentbalben ist das pathologisoho Gewebo reich an Corpora amylacea. Es wurde beschrieben, wie nach vorn die apoplectische Narbe, wie wir den Herd̉ nunmebr bezeichnen können, in die hintere Inselwindung ausläuft. Im Uebrigen erweist sich die gesammte Insel ebenso wie der Lobus Broca, die beide in fortlaufenden Schnittserien durchmustert wurden, völlig normal.

Im Stirnlappen fanden sich keinerlei Herderkrankungen; bei Zerlegung der Centralwindungen (links) in Schnitte, die senkrecht auf die Centralfurohe gelegt wurden, fand sich im Stabkranz der vorderen Centralwindung an der Gronze ihres oberen und mittleren Drittels, nahe der Decke des Ventrikels eine erbsengrosse Cyste. Innere Kapsel und Hirnschenkel frei von Degenerationen. In der rechten Brückenbälfte, Gegend des Abducensaustritts, wurde eine kaum hirsekorngrosse Cyste in einem der lateralsten Bündel der Pyramidenbahn entdeckt. Oblongata and Rückenmark intact; keinerlei secundäre Degeneration.

Die kleineren und mittelgrossen Gefässe des Gebirns, besonders im Bereich der centralen Ganglien, zeichnen sich durch Ablagerung von dunkelbraunem Pigment und ron körnigen Kalkconcrementen in der Adventitia aus; die Wandung erscheint dadurch auf Querschnitten von einem dunklen Ringe eingefasst; Ablagerungen ausserhalb der Gefässe und der Extravasate (Miliaraneurysmen) werden nicht aufgefunden.

Worttaubheit und Paraphasie, diese zwei Hauptkriterien der Wernicke'schen sensorischen Aphasie bildeten während der $3 \%$ jäh. rigen Beobachtung die constantesten und auffälligsten Erscheinungen in dem vorliegenden Krankheitsbilde. Die Worttaubheit besserte sich etwas im Laufe der Jahre; die Paraphasie machte sich geltend bei der Spontansprache, beim Nachsprechen, beim lauten Lesen, ganz besonders aber (wie gewöhnlich) bei der Schrift, sowohl bei der Spontanschrift, als beim Schreiben anf Dictat (das noch durch die Störungen des Wortverständnisses erschwert wurde). 
In völliger Uebereinstimmung mit der von Wernicke aufgestellten, von Naunyn bestätigten, Localisation steht der anatomische Befund: Herderkrankung im linken Schläfenlappen, vorzugsweise in Mark und Rinde der hinteren Zweidrittel der I. und der Randzone der II. Schläfenwindung. Wir müssen annehmen, dass an den sehr ausgeprägten paraphasischen Störungen auch die Betheiligung der Insel participirte. Die verbale Amnesie, die zu Anfang bestand, trat mehr und mehr zurück. Es ist dies ein gewöhnliches Verhalten; dasselbe gewinnt aber an Interesse durch die Thatsache, dass der Ausfall besonders Hauptworte, also Wortsymbole für concrete Begriffe betraf, dass damit ferner eine Schwierigkeit Hand in Hand ging, vorgẹzeigte Gegenstände richtig zu benennen, dass endlich gleichzeitig Andeutungen von Asymbolie vorlagen. Wir haben hier etwas vor uns, was Freund (l. c.) „optische Aphasie“ nennt, und werden kaum fehlgehen, wenn wir für diese Complication die Betheiligung des tiefen Marks des unteren Scheitelläppchens, das bis an das Ependym des Unter- und resp. Hinterhorns sklerosirt war, verantwortlich machen. - Uebrigens sei ausdrücklich hervorgehoben, dass ein dauernder Ausfall der Schriftbilder nicht bestand; literale Alexie war von Anfang an nicht vorhanden, aber auch von verbaler Alexie konnte, wenigstens später, wo noch Druckschrift correct copirt wurde, nicht die Rede sein. Auffällig erscheinen die Fehler im Lesen, besonders von zusammengesetzten Zahlen, während Patient mit den Zahlen im Ganzen richtig zu operiren vermag, um so auffälliger, als bei wirklicher Schriftblindbeit das Verständniss für Zahlen als Symbole für ganze Worte und nicht für Buchstaben besonders lange erhalten zu bleiben pflegt. - Nach der Anamnese möchte es scheinen, als ob Zeichen von Seelenblindheit (mangelhaftem Verständniss für gesehene Objecte) schon längere Zeit vor dem apoplectiformen Insult, mit dem die Aphasie einsetzte, bestanden hätten, schon zu der Zeit, in der der Kranke kritiklos Werthpapiere unter werthlose Schreiben mengte; die anatomische Untersuchung hat einen Anhaltspunkt für die Annahme verschiedenaltriger Herde indess nicht ergeben.

Die complicirende Seelenstörung steht in augenscheinlicher Continuität mit den aphasischen Störungen: das gestörte Wortverständniss, die Schwierigkeit sich zu orientiren, die Verkennung von Personen, das dunkele Gefühl der veränderten Persönlichkeit, zudem später, als bereits tiefere Störungen der Intelligenz vorlagen, die Verification von Träumereien - all das führte zu einer wahnhaften Auffassung der Umgebung in Form von Beeinträchtigungsideen. 
Die Integrität musikalischer Ausdrucksbewegungen (Melodien) bei unserem Kranken steht im Einklang mit ähnlichen Beobachtungen von Oppenheim (Charité-Annalen XIIL).

Im System würde unser Fall klinisch und anatomisch als Uebergangsform zwischen rein corticaler und subcorticaler sensorischer Aphasie eingereiht werden müssen (cfr. Malachowski, „Versuche einer Darstellung unserer hentigen Kenntnisse in der Lehre von der Aphasie“. Sammlungen klinischer Vorträge, heransgegeben v. Volkmann No. 324, 1888).

Herrn Dr. Tuczek, der mich bei dieser Arbeit wesentlich unterstützte, statte ich hiermit meinen innigsten Dank ab. 